\title{
Guest Editorial: Temporal representation and reasoning
}

\author{
Carlo Combi ${ }^{1}$
}

Published online: 14 June 2017

C Springer International Publishing AG 2017

\section{Introduction}

Temporal Representation and Reasoning has been an important topic and is orthogonal to many research areas in Computer Science. Besides being studied in many traditional and well established research areas as artificial intelligence, databases, real-time systems, and logics, time-related issues have been considered and studied even in the most recent research areas, as business process management, big data technologies, data mining and OLAP methods, and so on. As an example, in these recent years, data science emerged as a leading research trend. Nowadays, overwhelming amounts of data are produced in many different and heterogeneous contexts and need to be suitably represented, analyzed, merged, and queried, to derive significant information and knowledge. And even in this (relatively) new scenario, information is inherently temporal and needs to be properly managed, to derive trends and provide predictions, to manage time in new (big) data structures and languages.

Thus, we may say that theories, methodologies and techniques that explicitly focus on temporal features of information for representation and reasoning purposes are particularly important in many research topics of computer science and artificial intelligence. Among them, we mention here, without any claim to completeness: spatio-temporal and temporal databases; concurrent systems; temporal logics; knowledge discovery; knowledge representation and reasoning; big-data analytics; scheduling and planning; software security; and so on.

As a further consideration, Temporal Representation and Reasoning is a major theoretical and practical research area, as theoretical proposals need often suitable (methodologybased) tuning and extensions, to be applied to real world systems.

TIME Symposium aims to bring together researchers from distinct research areas involving the management of temporal data as well as the reasoning about temporal aspects of information. This unique and well-established event has as its objectives to bridge theoretical and

Carlo Combi

carlo.combi@univr.it

1 Department of Computer Science, University of Verona, Ca' Vignal 2 strada le Grazie 15, 37134 Verona, VR, Italy 
applied research, as well as to serve as an interdisciplinary forum for exchange among researchers from the areas of artificial intelligence, database management, logic and verification, and beyond. TIME has been for more than twenty years the only yearly multidisciplinary international event dedicated to the topic of time in computer science. Its purpose is to involve active researchers in different research areas considering temporal representation and reasoning. In the early years, most contributions came from the Artificial Intelligence community, but the number of contributions from other areas such as Temporal Logic and Verification and partly from Temporal Databases has been increasing in the last years. The TIME International Symposium Series began in 1994. It consists of annual meetings held mostly in Europe and sometime in North America. Over the years, participation of researchers from areas outside of mainstream AI has grown. For this reason, beginning with TIME'01, we have opened the meeting to active researchers in temporal, spatial, and spatio-temporal representation and reasoning from all areas. Also, a track format has been adopted, with artificial intelligence, databases, and logics, being the three main tracks.

The papers selected for this issue are deeply extended and revised versions of papers presented at TIME' 14 held on September 8-10, 2014, in Verona and, according to the depicted scenario, they cover a wide range of research issues spanning from spatio-temporal data modeling, to index structures for temporal data, to verification of concurrent systems, to metric temporal logics, up to variants for Computational Tree Logic.

\section{Content of the special issue}

This special issue contains five papers, with a wide range of research interests.

The first two papers, even though from different perspectives, focus on some issues in the area of temporal databases, more precisely on a framework for spatio-temporal data and its application, and on specific index structures for temporal databases, respectively.

Sara Migliorini and Alberto Belussi in their paper, entitled "A Spatio-Temporal Framework for Managing Archeological Data" propose a model, called Star, for representing spatio-temporal data in archeology, where vague representation is allowed by using fuzzy dates and fuzzy relationships among them. They also propose a set of rules for deriving temporal knowledge from the topological and stratigraphic relations existing between two archaeological findings. As a last contribution, they introduce the translation of archaeological temporal data into a Fuzzy Temporal Constraint Network for checking the overall data consistency and reducing the vagueness of some dates based on their relationships with other ones.

Fabio Grandi, in his paper, entitled "The RABTree and RAB-Tree: Lean Index Structures for Snapshot Access in Transaction-time Databases", introduces two lean temporal index structures to efficiently support snapshot access in a transaction-time database. The two proposed structures, the RABTree and its RAB-Tree variant, are conceptually simple, easy to implement and efficient secondary index solutions. Both theoretical and experimental evaluations of the two indexes are provided, in comparison with previously proposed indexing techniques.

The third paper is by Alejandro Sanchez and Cesar Sanchez and is entitled "Parametrized Verification Diagrams: Temporal Verification of Symmetric Parametrized Concurrent Systems" and deals with the verification of temporal properties of parametrized concurrent systems executed by an unbounded number of threads. The authors introduce parametrized verification diagrams (PVDs), which extend the generalized verification diagrams (GVDs) by adding support for parametrized verification. Parametrized verification diagrams allow 
using a single diagram to represent the proof that all possible instances of a given parametrized concurrent system satisfy the temporal specification considered. The presented PVDs cover a large class of concurrent and distributed systems, including concurrent data types. As a second contribution, the authors provide an implementation of PVDs and its integration into Leap, a prototype theorem prover. The practical applicability of PVDs, by using Leap, is then described by building and checking proofs of liveness properties of mutual exclusion protocols and concurrent data structures.

The last two papers deal with temporal logics. More particularly, Carlo Furia and Paola Spoletini, in their paper entitled "Bounded Variability of Metric Temporal Logic", deal with the issue of deciding validity of Metric Temporal Logic (MTL) formulas, by introducing bounded variability as a suitable restriction. Such a restriction limits the number of events possibly occurring over any time interval of a given length. The authors study the problem of deciding whether an MTL formula has intrinsic bounded variability, that is whether it is satisfied only by models with bounded variability. Finally, they also identify MTL fragments where deciding bounded variability is simpler than validity, possibly providing a reduction in complexity in some practical cases.

Finally, John McCabe-Dansted and Mark Reynolds, in their paper entitled "To be Fair, Use Bundles", deal with reasoning about systems with fairness properties. Fairness constraints are usually expressed as a restriction on paths through a transition structure. The authors show the usefulness and practicality of employing the bundled variants of CTLbased languages to handle fairness. In particular, the authors present a tableau for a bundled variant of CTL that still has the similar computational complexity to the CTL tableau and a simpler implementation.

\section{Putting together this special issue}

Putting together this special issue has been a long, interesting and intensive work. After the symposium, we invited the authors of six selected papers to submit an extended and revised version of their papers for inclusion in the special issue. For each submitted manuscript, two, and sometimes three and more,reviewers have been involved. A second, and for some papers even a third, revision and review phase followed for potentially accepted papers. Eventually, we selected five research papers.

We are indebted to many people for bringing this project to a successful conclusion. We thank Prof. Martin Golumbic, Editor-in-Chief of the journal Annals of Mathematics and Artificial Intelligence, for accepting our proposal of this special issue on temporal representation and reasoning. We thank all of the dedicated reviewers (a complete list of these follows) for their invaluable assistance in the production of this issue and for the interesting discussion we had with some of them. Finally, we thank the authors of all of papers submitted for this special issue.

REVIEWERS (in alphabetical order): Silvana Badaloni; Kristen Brent Venable; Eliseo Clementini; Curtis Dyreson; Francois Laroussinie; Jens Lechtenbörger; Ben Moszkowski; Gabriele Puppis; Karin Quaas; Ahmed Rezine; Pietro Sala; Marco Volpe; Robert Wrembel. 